\title{
In situ sampling uncovers the dynamics of particle genesis and growth in an aerosol tube reactor
}

\author{
Eric Bain Wasmund · Kenneth S. Coley
}

Received: 29 July 2005 / Accepted: 31 October 2005/Published online: 5 August 2006

(C) Springer Science+Business Media, LLC 2006

\begin{abstract}
Making metal and ceramic powders using aerosol synthesis from vapour precursors, either in a flame or hot-wall tube reactor, is the basis for producing many thousands of tons of powder on an annual basis. To properly study this system, we have designed and built a model reactor with sampling points at evenly spaced axial positions. This allows us to take snapshots of the aerosol population at many points within the reactor. Nucleation followed by a surface reaction produces a solid phase extremely rapidly, within $0.01 \mathrm{~s}$ under typical conditions. This is followed by a transient state where nucleation, surface reaction and coagulation all interact to produce a strongly bimodal size distribution. After nucleation is extinguished, the size distribution approaches the selfpreserving limit as predicted for a coagulation-dominated process. The final structure is determined by the dominant sintering mechanism, which can be estimated from theory. The knowledge of this mechanism offers the possibility of selecting reactor conditions to produce powders with optimized properties.
\end{abstract}

More than 100 years ago, Ludwig Mond and Carl Langer discovered that a number of transition metals react reversibly with carbon monoxide to form a class

E. B. Wasmund ( $\square)$

Inco Special Products, Mississauga, ON, Canada L5K 2L3

e-mail: ewasmund@inco.com

K. S. Coley

Department of Materials Science and Engineering, McMaster University, 1280 Main St., W. Hamilton, ON, Canada L8S 4L7 of compounds known as the metal carbonyls [1]. In these compounds, $\mathrm{CO}$ molecules are bound as ligands to the metal. They typically exist as vapour under ambient conditions, and are generally quite toxic. The equilibrium for dissociation of nickel carbonyl is shown as Eq. 1 [2].

$\mathrm{Ni}(\mathrm{CO})_{4} \leftrightarrow \mathrm{Ni}(\mathrm{s})+4 \mathrm{CO}$
$\Delta G^{\mathrm{o}}(\mathrm{kJ} / \mathrm{mol})=155.20-0.3964 T(\mathrm{~K})$

From Eq. 1, nickel carbonyl is favoured by lower temperature and higher pressure. The discovery of these compounds paved the way for industrial innovations in the areas of extractive metallurgy and chemical vapour deposition [3].

Regarding extractive metallurgy, industrially significant processes for the low-cost purification of nickel by the carbonyl process have been in continuous operation since 1902. In these processes, metals that have the tendency to form carbonyls are readily separated from other metals by passing carbon monoxide through a bed of the finely divided feed, under conditions that favour the left-hand side of Eq. 1. Metals such as nickel will selectively form gaseous nickel carbonyl, leaving other metals such as copper, behind in the bed. The nickel carbonyl can then be deposited as pure nickel by raising the temperature.

Regarding chemical vapour deposition (CVD), metal carbonyls can be been used as CVD precursors to make a variety of materials such as nickel-coated fibres, foam and powders [4]. In these processes, metals are deposited heterogeneously from the gas-phase onto a heated template, again using the reaction described in Eq. 1. The kinetics for the heterogeneous reaction 
have been well studied and can be accurately described by a second-order mechanism [5]. As a result, an adequate application of chemical kinetics, heat transfer and mass transport allows practitioners to reliably deposit metal coatings with precise control of thickness and microstructure.

The metal carbonyls can also be used to make powders by free-space decomposition. Iron and nickel powders have been made by this process since the 1920's using flow-through tube reactors developed originally by Alwyn Mittasch [3]. In these reactors, the metal carbonyl, in a suitable carrier gas such as carbon monoxide is fed into the top of a tube, typically 1-2 m in diameter and $5-10 \mathrm{~m}$ in height. The tube is heated through the walls, and as the metal carbonyl descends through the reactor it reaches a critical temperature, which initiates the decomposition reaction, resulting in the nucleation of a solid phase and liberation of CO. These particles continue to grow by a surface reaction as they are carried by gravity and gas entrainment to the bottom of the reactor where they are collected in a hopper.

The science that defines the control of particle properties in this process is much more complicated than chemical vapour deposition alone. Equation 1 can proceed by two parallel pathways, a homogeneous decomposition, or nucleation reaction, and a heterogeneous reaction on the surface of pre-existing particles. As these reactions occur, the particles can also grow by collision. This is known as coagulation in the aerosol literature, and the final particle structure can also be affected by gas-phase sintering. Besides metal carbonyl decomposition, aerosol material synthesis processes are also used to produce many other types of finely divided powders such as pigment grade titania, fumed silica, and carbon black [6]. In many of these cases, nucleation, surface reaction, coagulation and sintering have been studied in isolation, but how they interact in the free space of an aerosol tube reactor is not generally understood. Detailed knowledge of the fundamentals would allow industrial practitioners to develop new powder products and to produce powders with optimized properties.

\section{Experimental}

To understand the details of this process, what is happening inside the black-box, we have built a model reactor that can be interrogated to provide detailed "snap-shots" of the particle population at many points during it's trajectory. For the first time ever, the details of the nickel and iron carbonyl powder making process can be studied and compared with models based on first principles. Our system consists of a $5 \mathrm{~cm}$ diameter tube that is $25 \mathrm{~cm}$ in height with five sampling arms perpendicular to the flow located axially every $5 \mathrm{~cm}$. A photograph of the reactor is shown as Fig. 1. In this reactor, the carbonyl-laden feed gas is fed through a water-cooled nozzle into the top of the reactor. The furnace consists of five independently controlled heating zones that conduct heat through the reactor wall. The particles are collected in a filter bag at the bottom of the reactor.

For the experiment described here, 3 standard litres/ min of feedgas, comprised of $5 \mathrm{vol} \%$ nickel carbonyl and $330 \mathrm{ppm} \mathrm{NH}_{3}$ in a balance of carbon monoxide was fed into the top of the reactor. The outside wall temperatures were maintained at about $600{ }^{\circ} \mathrm{C}$ and the system pressure was $1 \mathrm{psig}$. The internal temperatures, measured by in-situ temperature measurements are shown in Table 1. The top sampler was deployed for $150 \mathrm{~ms}$ and the remaining four samplers were each deployed for $1000 \mathrm{~ms}$. Two minutes elapsed between each deployment to allow the system to return to steady state.

Each sampling arm contains a cylindrical sampler with two flattened edges, which have been each fitted with three holders containing standard transmission electron microscope (TEM) grids. The foreground of Fig. 2 shows one of the five samplers used in this study. The inset panel shows one of the carbon-coated copper TEM grids used on each sampler. The mechanism of particle deposition onto the grids is thermophoresis, this method has been used by a number of researchers elsewhere [7, 8]. A thermophoretic force acts on the hot aerosol particles and pushes them towards cold surfaces [9]. This is an ideal method of capturing particles since it is essentially independent of particle size

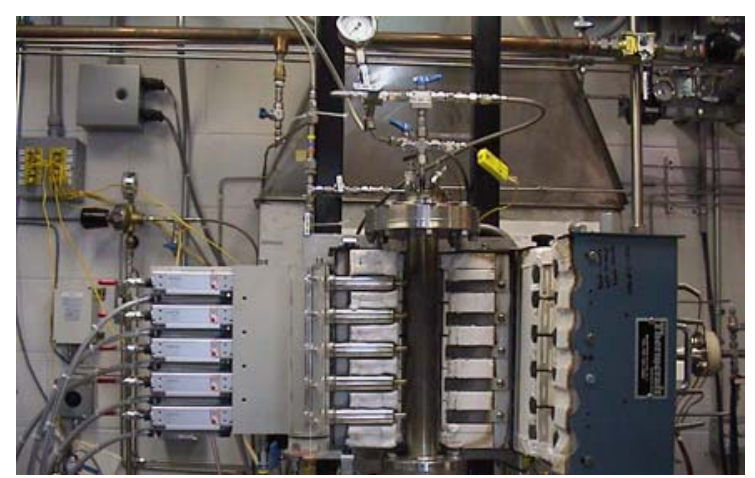

Fig. 1 Close-up of the $5 \mathrm{~cm}$ diameter tube reactor with the electrical heaters pulled away. On the left-hand side are the water jacketed arms and the pneumatic pistons that sample into the reactor 
Table 1 The residence time and temperature, from CFD analysis, and in-situ sampling respectively, for the reactor centre-line position of each sampler

\begin{tabular}{llll}
\hline Sampler & \multicolumn{3}{l}{$\begin{array}{l}\text { Internal states within the reactor corresponding to } \\
\text { sampler positions }\end{array}$} \\
\cline { 2 - 4 } & $\begin{array}{l}\text { Distance } \\
\text { from inlet }[\mathrm{cm}]\end{array}$ & $\begin{array}{l}\text { Residence time } \\
\text { from inlet }[\mathrm{s}]\end{array}$ & $\begin{array}{l}\text { Temperature } \\
{\left[{ }^{\circ} \mathrm{C}\right]}\end{array}$ \\
\hline 1 & 5 & 0.01 & 350 \\
2 & 10 & 0.08 & 410 \\
3 & 15 & 0.37 & 430 \\
4 & 20 & 0.73 & 460 \\
5 & 25 & 1.11 & 450 \\
\hline
\end{tabular}

when the particles are smaller than the mean-free path of the gas, and when they are not, the decreased mobility can be estimated using well defined expressions such as Talbot's equation [9]. The deployment of the samplers will now be described.

Each sampler resides in a sampler side-arm, shown in Fig. 1. In the un-deployed state, the sampler is isolated from the free space of the reactor. A small flow of inert gas is bled through the sample arm to insure that the reactor contents cannot come in contact with the sampler, except during deployment. Each sampler side-arm is also cooled by an annular water-cooled jacket, shown in Fig. 1, which insures that there is an adequate temperature difference between the grids and the aerosol to drive the thermophoretic deposition. The objective is to operate the samplers in a regime where thermophoresis was at least ten times more significant than Brownian diffusion, thus avoiding a deposition mechanism that would bias the population [9]. Each sampler was coupled to a high-speed doubleaction pneumatic piston. The timer/actuator for the pistons allowed for a precise deployment of each

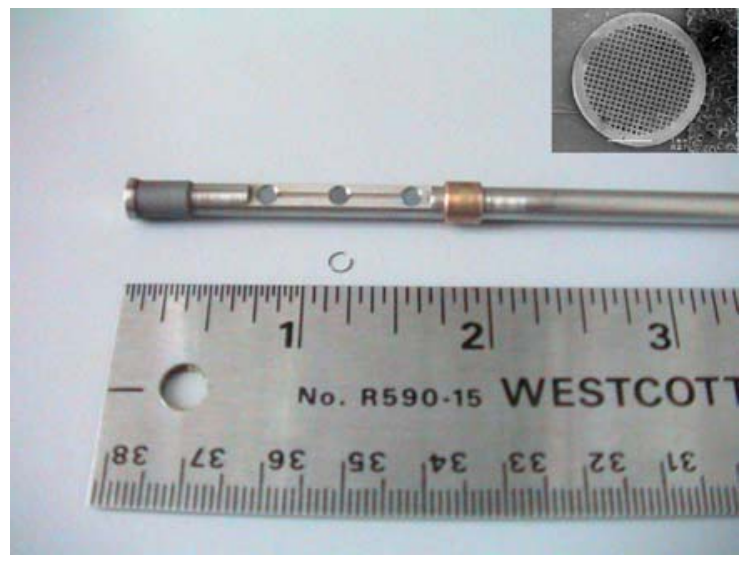

Fig. 2 One of the thermophoretic samplers used in this study. Inset: a low magnification micrograph of one of the sample grids used in this study sampler, with approximately $20 \mathrm{~ms}$ of travel time into and out of the reactor. By this method, the sample grids could be inserted into the reactor parallel to the direction of convective gas flow and "exposed" for precisely controlled periods of time.

\section{Results and discussion}

The particle populations at the centre-line for each sampler are shown as Fig. 3. Figure 3a shows a scanning transmission electron microscope (STEM) (JEOL 2010F) micrograph of samples taken from the centreline of the first sampler $(5 \mathrm{~cm}$ from the inlet $)$. This picture reveals the presence of a relatively monodisperse population of very fine particles, with an average size of $50 \mathrm{~nm}$. No large particles were detected on the sample grid. Figure $3 \mathrm{~b}$ and $\mathrm{c}$ show micrographs from the second sampler, observed at magnifications of $5,000 \times(\mathrm{SEM})$ and $60,000 \times(\mathrm{STEM})$, respectively. These images suggest that there is a bimodal population at the position of the second sampler. Figure $3 \mathrm{~d}$ and $3 \mathrm{e}$ show low magnification images from the third and fourth sampler. High magnification images from these samplers, not shown here, reveal that there are no longer any particles less than $50 \mathrm{~nm}$ after the second sampler.

Computational fluid dynamics is a mathematical method for calculating the velocity, concentration and temperature profiles by dividing the geometry of interest into a number of adjacent cells and then solving discretized versions of the conservation equations for each cell. The technique has been used extensively to understand the temperature and flow patterns in aerosol reactors, assuming in most cases that the particle phase is sufficiently dilute to have any effect on the fluid states [10]. A detailed computational fluid dynamic model of this system was also developed to estimate the residence time of the gas at the centreline [11]; shown in Table 1. Because of the small size of the particles in Fig. 3, they will effectively track the bulk flow of the gas, in other words, they will have the same residence time distribution as the gas in this reactor.

Ten micrographs of the type shown in Fig. 3 were analyzed by image analysis to derive population statistics such as number-weighted size distributions for each of the five samplers at the centre-line. For segments of the distributions representing particles greater than the mean-free path of the gas, a correction was applied, using Talbot's equation to account for the decreased mobility (and hence observed frequency) of larger particles. Then the distributions were 


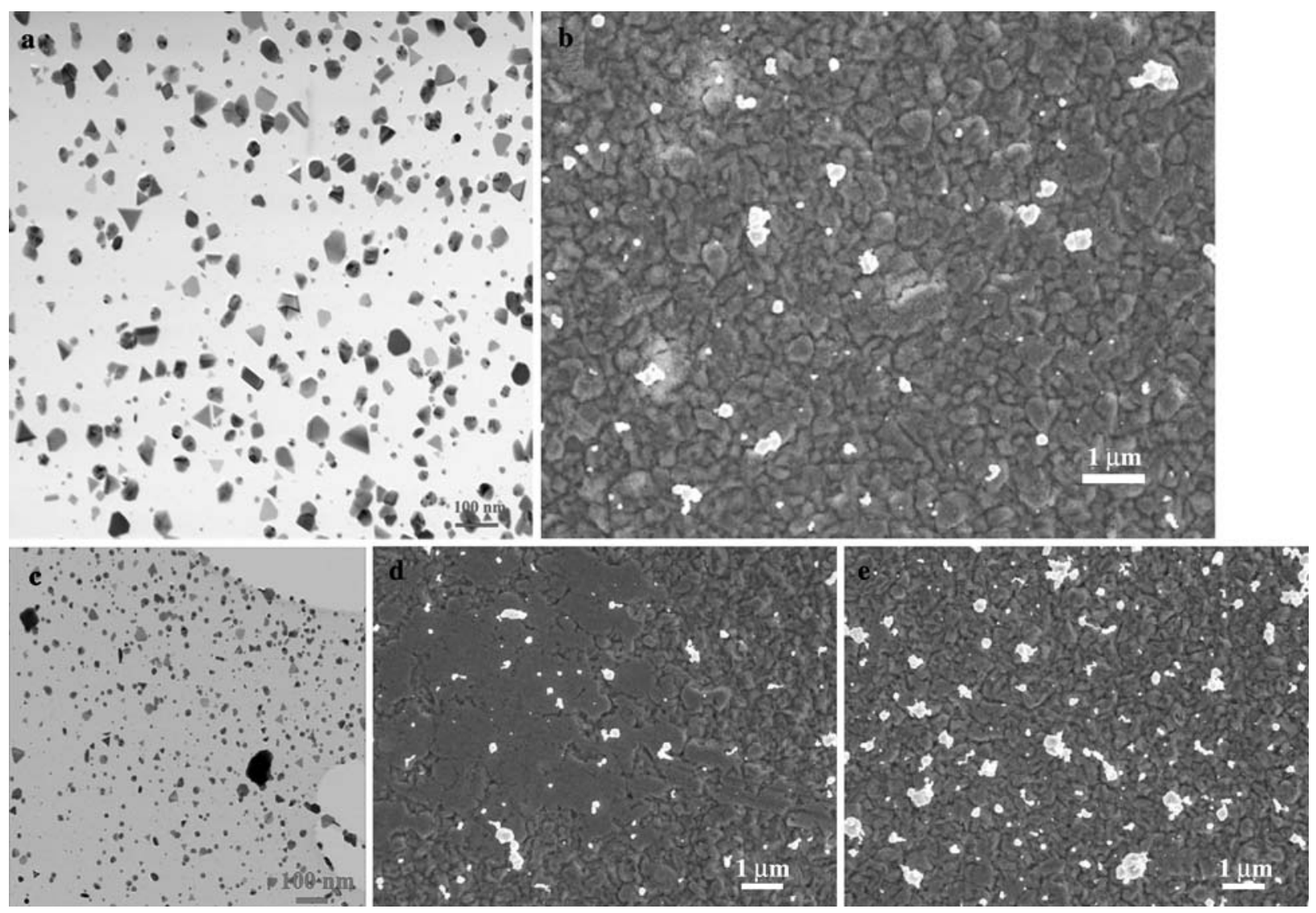

Fig. 3 Representative images from along the centre-line of the experiment. (a) The particles formed $5 \mathrm{~cm}$ from the inlet (residence time $0.01 \mathrm{~s}$ ) by STEM. (b) The particles at $10 \mathrm{~cm}$ from the inlet (residence time $0.08 \mathrm{~s}$ ) by SEM. (c) The particles

transformed from number-weighted to volume-weighted by scaling each segment in the distribution by the corresponding average volume. Using standard statistical equations [12], the volume-weighted distributions could be used to calculate the geometric mean diameter (GMD) and geometric standard deviation (GSD), assuming that the population can be reasonably described by a log-normal relationship. In a log-normal distribution, the GMD is the average size and the GSD is a measure of the spread, or polydispersity of the distribution. The resulting picture of the evolving aerosol population for this experiment is shown as Fig. 4.

In addition to estimates of the size distribution, estimates of the particle number concentration at each sampling position in the reactor can also be calculated. The thermophoretic velocity can be calculated using Talbot's equation, and the frequency of particles that have collected on the sample grids can also be measured. This data can then be combined to give estimates of the particle number concentration in the aerosol stream. at $10 \mathrm{~cm}$ from the inlet under high magnification by STEM. (d) The particles at $15 \mathrm{~cm}$ from the inlet (residence time $0.37 \mathrm{~s}$ ) by SEM. (e) The particle at $20 \mathrm{~cm}$ from the inlet (residence time $0.73 \mathrm{~s}$ ) by SEM

Figure 4 shows some very interesting features that were corroborated by 20 other experiments using the same apparatus [11]. The homogeneous reaction, or nucleation step occurs very early in the process, at a much lower temperature than predicted by classical nucleation theory. Within $0.01 \mathrm{~s}$, approximately $10^{15}$ particles per gram of gas appear that have consumed about $70 \%$ of the starting nickel carbonyl [11]. High resolution TEM confirms that these particles are single crystals, suggesting that these particles have grown mainly by a surface reaction, and not coagulation, which would have produced randomly oriented polycrystals.

The most interesting behaviour of the system occurs between 0.01 and $0.08 \mathrm{~s}$. During this time, the $50 \mathrm{~nm}$ particles have begun to combine by coagulation, this is expected to happen very quickly since the rate of Brownian coagulation is proportional to the square of the number concentration. The nucleation reaction has not been completely disabled, so that smaller particles concurrently form and enter the distribution from the 
Fig. 4 Mass weighted histograms, based on image analysis of micrographs collected from the samplers. After $0.01 \mathrm{~s}$, the distribution is narrow with all particles less than about $100 \mathrm{~nm}$. At $0.08 \mathrm{~s}$, the distribution has become strongly bimodal as nucleation, surface reaction and coagulation all co-exist. Some time after $0.08 \mathrm{~s}$, nucleation is extinguished and particle growth continues by the surface reaction and coagulation. As coagulation dominates, the spread of the distribution approaches the self-preserving limit

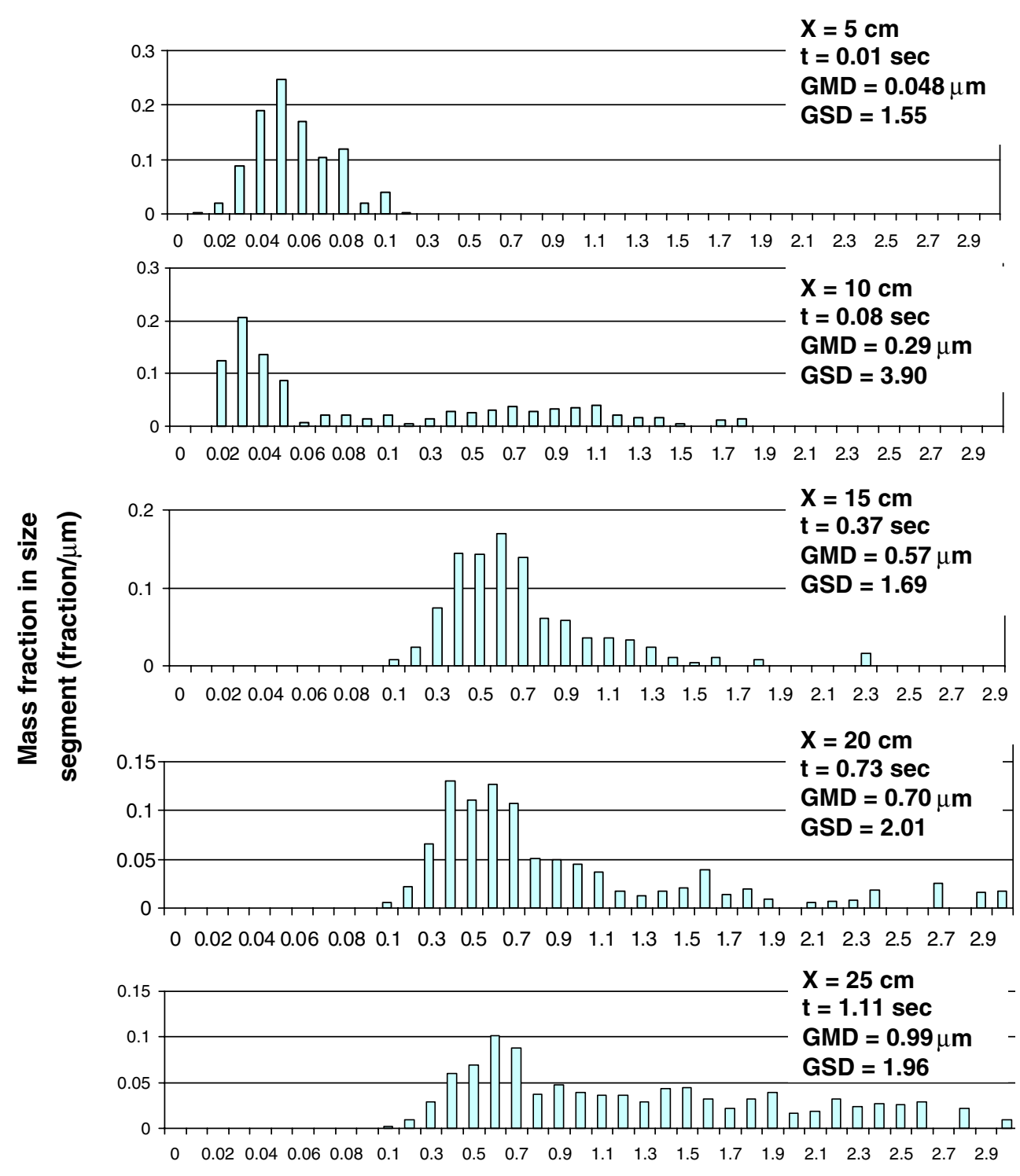

Particle size $(\mu \mathrm{m})$ left-hand side of the histogram in Fig. 4. They grow more slowly than the particles observed at the first sampler because of the low nickel carbonyl concentration, resulting in a finer size. The simultaneous action of nucleation, surface reaction and coagulation have produced a transient bimodal distribution with a maximum polydispersity of GSD $=3.90$. The particle concentration is still extremely high, approximately $10^{15}-10^{16}$ particles per gram of gas.

By the position of the third sample, corresponding to a residence time of $0.37 \mathrm{~s}$, nucleation, as observed by the presence of very fine particles, has been extinguished and the particles continue to grow mainly by Brownian coagulation. The particle concentration has decayed to approximately $10^{11}-10^{12}$ particle per gram of gas. As the particles continue down through the reactor without any new reactant, they continue to grow by collisions, and the spread of the distribution approaches a constant value, which is expected for a coagulation-dominated process [9]. The evolution of the size distribution to a constant value of polydispersity can be observed by plotting the GSD of the centreline volume size distribution against the residence time. This is shown for two experiments conducted under these same conditions in Fig. 5.

This picture from inside the aerosol reactor suggests fast nucleation of particles, followed by a period of simultaneous nucleation, surface reaction and coagulation in which the spread of the size distribution reaches a maximum, followed by coagulation only, and 
the movement towards a constant GSD or spread of the distribution with time. This phenomenon was previously predicted mathematically for the oxidation of $\mathrm{TiCl}_{4}$ in an aerosol tube reactor using a sectional aerosol model $[13,14]$. Both of these groups showed that the polydispersity starts off at a minimum as the reaction begins by nucleation and surface reaction, then goes through a maximum as a result of a temporary bimodal distribution, when nucleation, surface reaction, and coagulation all co-exist. During the temporary bimodal distribution, particles being produced at the fine end of the distribution are smaller than particles produced during the initial stages of the process. Finally the population approaches the limiting value of the polydispersity as the distribution approaches the self-preserving limit, which is characteristic for aerosol systems growing by Brownian coagulation only. A simplified model of the aerosol dynamics for this system has been developed, which includes nucleation, surface reaction and coagulation, and the model predictions have been compared with the distributions obtained by sampling along the centre-line. The model, which assumes that the size distribution of particles is monodisperse, is in reasonable agreement with the experimental data when the population of particles is roughly log-normally distributed [11].

It should be noted that the plateau versus time of the GSD observed in these experiments is somewhat higher than the value of the GSD calculated for the self-preserving limit. Under the conditions of these experiments, the Hausdorff parameter $D_{\mathrm{f}}$, was measured to be about 2.0. Vemury and Pratsinis [15] have

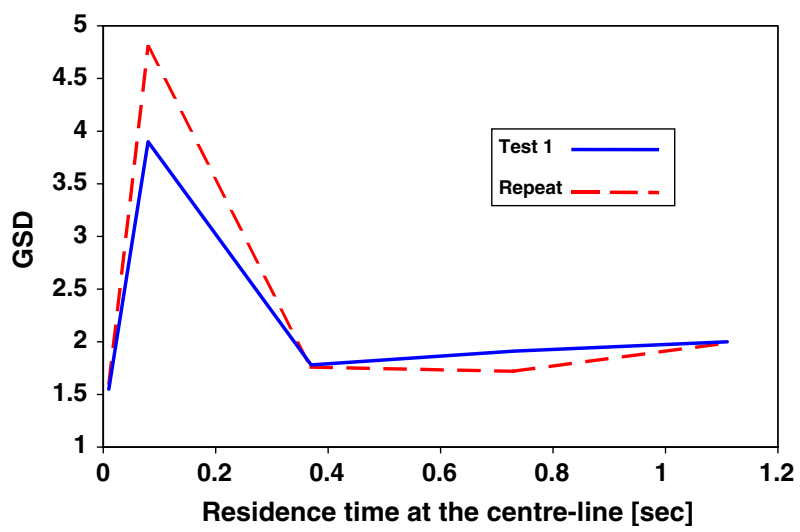

Fig. 5 The experimental geometric standard deviation (GSD) at the centre-line for the volume distribution of two experiments conducted under the conditions reported in this study. Nucleation and surface reaction create initial particles with a low GSD, then the continuation of these processes in the presence of coagulation creates a maximum, which slowly moves towards an asymptotic value as a result of coagulation alone performed simulations for non-spherical agglomerates to show that under these conditions, the GSD of the volume distribution in the continuum regime should be about 1.30. These simulations were made for an ideal ageing aerosol with no convective mixing, and so the deviation between our experimental results and the simulated value is probably a combination of fluid mixing in the reactor and experimental error in the collection and measurement of aerosol populations.

The consequences of these observations can be significant for practitioners trying to make metal powders with optimized properties. For example, in many applications, powders with a narrow size distribution are preferred. According to our results, the process is dominated by Brownian coagulation after 0.1 seconds. After this point, the minimum polydispersity or GSD of the population is fundamentally limited as the "selfpreserving size distribution" is approached. Of course, the actual polydispersity can be higher, depending on the degree of fluid mixing in the reactor.

One of the interesting implications of this view within the reactor, is that sintering of the particles must be very rapid, in fact the particles that come out of the bottom of the reactor, Figure $3 e$ do not seem to be comprised of the fine particles shown in Fig. 3a. To prove that this can be accounted for by coagulation and sintering, the time constants for particle sintering by a variety of mechanisms were calculated for nickel using the 2-sphere model [16]. This model provides an estimate for the sintering time of two equi-sized particles. Many of the initial particles consist of platelets and should sinter very effectively. At the prevailing internal temperature and residence time of this study, approximately $440{ }^{\circ} \mathrm{C}$ and $1 \mathrm{~s}$, the maximum particle size for initial sintering by surface diffusion, evaporation-condensation, grain boundary diffusion and volume diffusion is $0.32,0.005,0.17$ and $<0.001 \mu \mathrm{m}$ [11]. Because the largest quasi-spherical particles are approximately $0.7 \mu \mathrm{m}$, the conclusion is that the predominant mechanism for sintering under these conditions is surface diffusion, the temperature is too high for significant evaporation-condensation via a carbonyl intermediary and it is too low for grain-boundary or volume diffusion.

High-resolution field emission scanning electron microscope (SEM) images of typical particles at each sampler suggest that the particles have grown by coagulation and sintering, shown in Fig. 6. Figure 6a shows the plate-like morphology of many of the very fine particles produced after $0.01 \mathrm{~s}$. Figure $6 \mathrm{~b}$, a typical particle after $0.08 \mathrm{~s}$ suggests that a 0.5 micron particle has grown by the accumulation of many of the smaller particles present earlier. Further displacement through 
Fig. 6 Typical particles at the centre-line as viewed by highresolution field emission SEM. (a) At $5 \mathrm{~cm}(0.01 \mathrm{~s})$, nucleation is much faster than coagulation. (b) At $10 \mathrm{~cm}$ $(0.08 \mathrm{~s})$ smaller particles have collided together and melded by surface diffusion. (c) $(15 \mathrm{~cm}, 0.37 \mathrm{~s}),(\mathbf{d})(20 \mathrm{~cm}$, $0.73 \mathrm{~s}$ ). As the residence time increases, samples show less detail that reveal their genesis
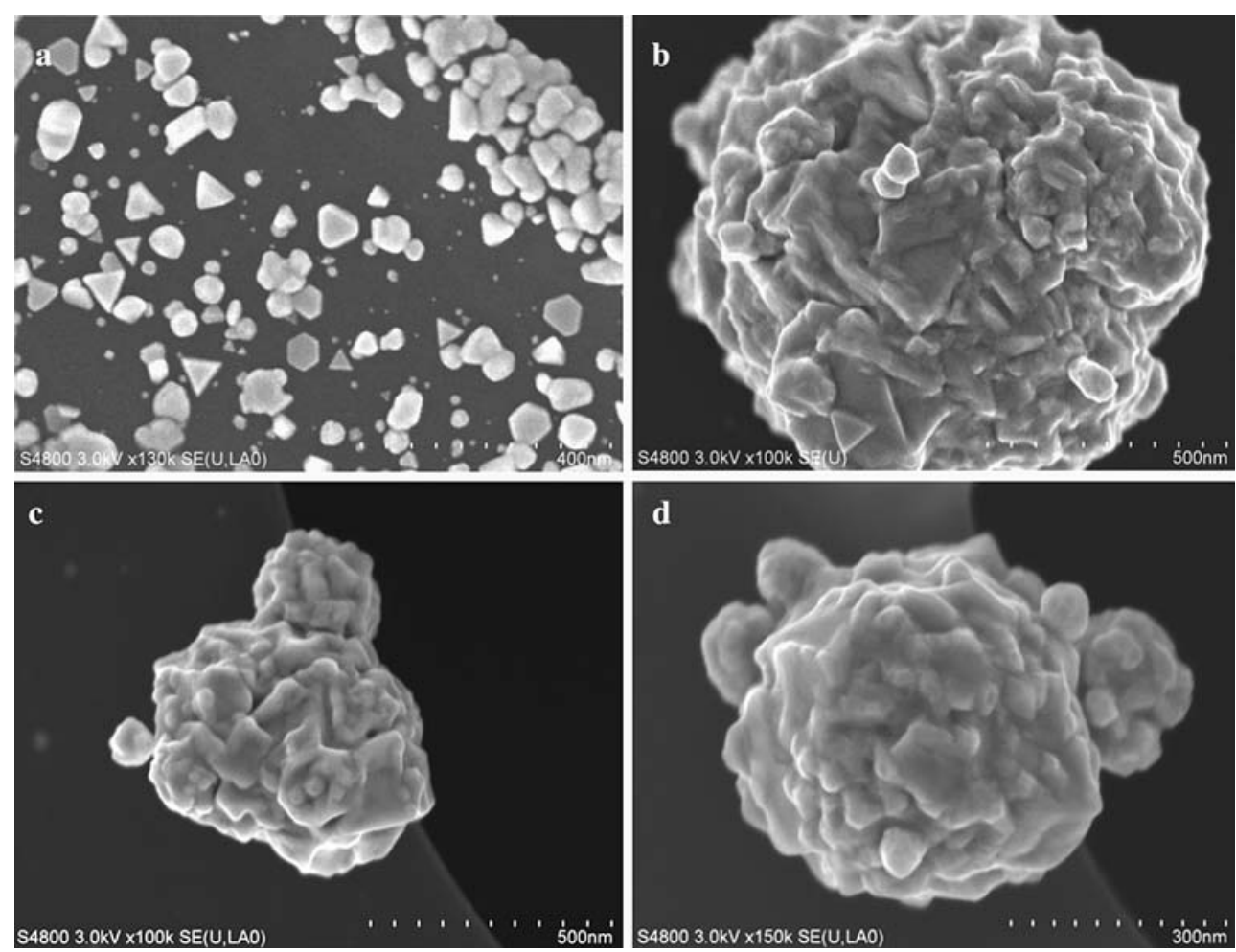

the hot reactor shows a progressive smoothing of the particle surface ( $6 \mathrm{c}$ corresponds to $0.37 \mathrm{~s}$ and $6 \mathrm{~d}$ corresponds to $0.73 \mathrm{~s}$ ). Near the bottom of the reactor, the surface has been sufficiently smoothed and it is no longer obvious how the particle has grown.

As final proof of this mechanism, a focused-ion beam was used to section a typical particle. The particle was subsequently imaged in back scattered electron (BSE) mode to contrast the crystallographic orientation inside the particle-this image is shown in Fig. 7. The exterior of the particle shown in Fig. 7 is smooth, suggesting significant surface sintering, however on the inside, there are crystals, approximately $100 \mathrm{~nm}$, the same order of magnitude as the fine particles produced in the top of the reactor (Fig. 3a). There is no observed orientation relationship between adjacent crystals inside the particle, which is consistent with growth by coagulation.

\section{Summary}

Specific engineering of the material properties of aerosol-produced powders becomes a possibility when each step of the process is understood from the perspective of the scientific fundamentals. This deep process knowledge has become available for the metal carbonyl systems through the combination of in-situ sampling and CFD analysis, the "black-box" has finally been opened up. For the temperature ranges used in this process, the crystal size is determined by nucleation and surface reaction, which occurs very early in the overall process. The smoothness of the particle surface is controlled by surface sintering, evaporationcondensation below about $300^{\circ} \mathrm{C}$ or surface diffusion above this temperature. Finally, the size and shape of the size distribution are controlled by coagulation. The

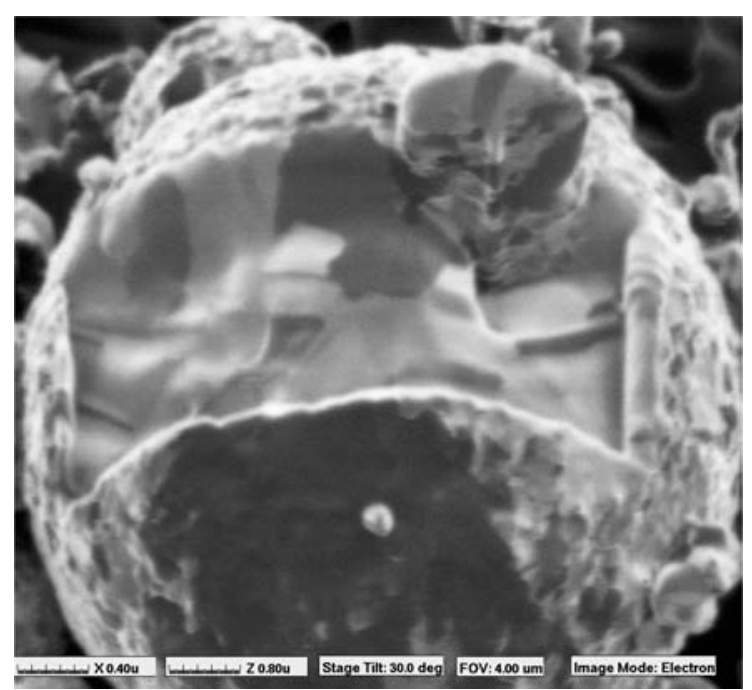

Fig. 7 A focused ion beam section of a nickel particle made from nickel carbonyl, revealing details of the internal crystal structure 
spread of the distribution has a lower limit, which can be calculated from aerosol physics. The dynamic evolution of the size distribution was shown to be qualitatively in agreement with mathematical models developed for other aerosol synthesis processes. It is expected that many of these insights will carry over to other aerosol synthesis systems.

Acknowldegements Josef Benedik from Pero Inc designed and built the thermophoretic sampling system. Dave Hoyle at Hitachi High-Technologies Canada Inc was responsible for the FESEM work. Mike Phaneuf at FIBICS Incorporated was responsible for the FIB imaging. Fred Pearson at McMaster University was responsible for the STEM work. Randy Shaubel from Inco Technical Services Limited ran the experiments.

\section{References}

1. Mond R (1930) J Soc Chem Ind XLIX(24):271, XLIX(25):283, $\operatorname{XLIX}(26): 287$

2. Barin I (1989) Thermochemical data of pure substances, $\mathrm{VCH}$
3. Trout W (1938) J Chem Educ March:113

4. www.incosp.com

5. Carlton H, Oxley J (1965) Am Inst Chem Eng J 11(1):79

6. Pratsinis S, Vemury S (1996) Powder Technol 88:267

7. Dobbins R, Megaridis C (1987) Langmuir 3:254

8. Arabi-Katbi O, Pratsinis S, Morrison P, Megaridis C (2001) Combust Flame 124:560

9. Friedlander S (2000) Smoke, dust and haze, 2nd edn. John Wiley

10. Kammler H, Madler L, Pratsinis S (2001) Chem Eng Technol 24:583

11. Wasmund E (2005) PhD Thesis, Department of Materials Science and Engineering, McMaster University

12. Hinds W (1999) Aerosol technology: properties, behavior, and measurement of airborne particles, 2nd edn. John Wiley and Sons

13. Xiong Y, Pratsinis S (1991) J Aerosol Sci 22(5):637

14. Tsantilis S, Pratsinis S (2000) Am Inst Chem Eng J 46(2):407

15. Vemury S, Pratsinis S (1995) J Aerosol Sci 26(2):175

16. German R (1996) Sintering theory and practice. John Wiley 